\title{
Les données entre normalisation et territoire : la construction de la trame verte et bleue
}

Naturalist data between standards and territory : building ecological network (TVB) in France

Pierre Alphandéry, Agnès Fortier et Anne Sourdril

\section{(2) OpenEdition}

\section{Journals}

Édition électronique

URL : http://journals.openedition.org/developpementdurable/9282

DOI : 10.4000/developpementdurable.9282

ISSN : 1772-9971

Éditeur

Association DD\&T

\section{Référence électronique}

Pierre Alphandéry, Agnès Fortier et Anne Sourdril, « Les données entre normalisation et territoire : la construction de la trame verte et bleue », Développement durable et territoires [En ligne], Vol. 3, n 2 | Juillet 2012, mis en ligne le 12 juillet 2012, consulté le 19 avril 2019. URL : http:// journals.openedition.org/developpementdurable/9282; DOI : 10.4000/developpementdurable.9282

Ce document a été généré automatiquement le 19 avril 2019

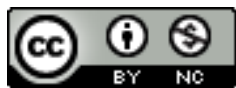

Développement Durable et Territoires est mis à disposition selon les termes de la licence Creative Commons Attribution - Pas d'Utilisation Commerciale 4.0 International. 


\section{Les données entre normalisation et territoire : la construction de la trame verte et bleue}

Naturalist data between standards and territory : building ecological network (TVB) in France

Pierre Alphandéry, Agnès Fortier et Anne Sourdril

1 La Trame Verte et Bleue (TVB) nationale adoptée lors du Grenelle de l'environnement, en 2007, a pour ambition de lutter contre la fragmentation des habitats naturels engendrée par les activités humaines, en instaurant des continuités écologiques entre les réservoirs de biodiversité afin de favoriser la circulation des espèces et le brassage génétique des populations. Comme toutes les politiques de conservation de la biodiversité, la TVB s'appuie sur des connaissances scientifiques tout en s'efforçant de concilier les enjeux politiques et sociaux. Mais selon le Ministère de l'écologie, la trame doit en outre « offrir l'opportunité d'une approche intégrée d'aménagement du territoire et de préservation des milieux naturels $»^{1}$. Elle apparaît, dès lors, comme un point de rencontre d'enjeux écologiques et territoriaux, tout en laissant ouvertes les modalités d'implication des groupes locaux et leur interaction avec les dimensions scientifiques. C'est précisément cette question des rapports entre science et société que nous souhaitons interroger à travers le processus d'identification des réseaux écologiques à l'échelle des territoires (Région, Parcs Naturels Régionaux (PNR), groupements de communes) qui constitue la première étape de la mise en œuvre de la TVB.

2 La phase d'identification est décisive comme nous l'avons vu à propos de l'application de la directive Habitats en France (Rémy et al., 1996). Elle met en jeu des connaissances expertes, des savoirs localisés, des identités diverses, des pratiques de l'espace, des rapports entre le local et le global fondés sur une rhétorique de défense du vivant et, enfin, elle s'appuie sur un dispositif articulé sur trois niveaux d'échelle: national, régional, local. Dans ce processus d'agencement complexe, les connaissances mobilisées jouent un rôle déterminant. Qu'elles soient écologiques ou territoriales, quantitatives ou 
qualitatives, répertoriées ou non dans des banques de données, ces diverses formes de connaissances apparaissent comme autant d'outils susceptibles d'être mobilisés pour identifier les réservoirs de biodiversité, les corridors et au-delà les objectifs assignés à cette trame : écologiques mais aussi culturels, paysagers, économiques, etc. La question en jeu ici n'est pas tant de réfléchir sur le bien-fondé ou la pertinence de telle ou telle méthode d'identification des réseaux écologiques mais elle vise plus fondamentalement à s'interroger sur qui participe, au moyen de quels instruments de connaissance et pour faire valoir quels enjeux. Comme le soulignait la Sénatrice Verte M.-C. Blandin lors du congrès de France Nature Environnement (FNE) consacré à la TVB (2009), «La trame n'est pas seulement un but en soi (...). Il s'agit d'une opportunité pour que des gens qui ne se parlent pas d'habitude se rencontrent, échangent leurs buts, leurs connaissances, leurs contraintes et arrivent à accoucher de quelque chose ». Dans un contexte marqué par la montée de l'expertise (Demortain, 2012 ; MEDDTL, 2011 ; Berrebi-Hoffmann et Lallement, 2009), nous faisons l'hypothèse que cette intégration de tous les acteurs, officiellement recherchée, ne peut se faire que si sont réunies les conditions d'un véritable dialogue autour des connaissances d'origines diverses. L'exemple de Natura 2000 a, en effet, montré que l'identification des sites par les seuls experts naturalistes a conduit au blocage du processus de mise en œuvre, lequel a pu être levé grâce à une procédure de concertation impliquant les acteurs locaux (Alphandéry et Fortier, 2007b). A partir d'une expérience conduite au sein d'un PNR, nous questionnerons les types de données mobilisés pour identifier les continuités et leur complémentarité, les acteurs qui les portent et les espaces de débats mis en place. L'enquête s'appuie sur un travail d'observation participante (comités de suivi du projet) et la réalisation d'entretiens semidirectifs auprès des participants. Elle a permis de mettre au jour les différents types de connaissances convoqués et d'observer in situ les interactions entre experts naturalistes et experts locaux.

Dans la première partie de cet article, nous rappellerons l'ambition de la TVB, le dispositif prévu pour sa mise en œuvre et la dimension cognitive qui est au centre de sa construction. La seconde partie sera consacrée à une étude de cas qui s'attachera à mettre en évidence la difficulté à combiner différentes formes de connaissances et d'outils dans le processus d'identification des corridors écologiques. Dans une troisième partie, nous reviendrons sur la diversité des formes de savoirs mobilisés et leur plus ou moins grande légitimité, ce qui nous conduira à examiner les rapports souvent étroits entre les questions cognitives et politiques.

\section{La TVB entre écologie et territoire}

4 Considérée comme une des mesures phares du Grenelle de l'environnement, la TVB est aussi l'aboutissement d'un processus engagé en 1995, lors de la ratification par la France de la Stratégie paneuropéenne pour la diversité biologique et paysagère qui prévoit la mise en place d'un réseau écologique sur le continent (Fabos et Ahern, 1996). Inscrit dans la loi Grenelle 1 (2009), le projet de TVB intervient à l'issue d'une période de plus de 15 ans marquée par la mise en œuvre conflictuelle de la directive Habitats (Alphandéry et Fortier, 2007b). Il répond également à la recherche d'une politique innovante en France, au moment où la tentative de stopper l'érosion de la biodiversité en France et dans l'UE en 2010 (année internationale de la biodiversité) s'avère devoir rester hors de portée 
selon les propos exprimés par les responsables de la Commission et de l'administration hexagonale.

Fondée sur une approche fonctionnelle des systèmes écologiques, la TVB incarne un renouvellement dans les manières de lutter contre la disparition du vivant. Mais au-delà de ces considérations, le projet de TVB élaboré conjointement par des acteurs scientifiques et non scientifiques dans le cadre du Grenelle, doit constituer un outil d'aménagement du territoire et « mettre en synergie les différentes politiques publiques afin de maintenir ou de restaurer les capacités de libre évolution de la biodiversité au sein des territoires, notamment en maintenant ou en rétablissant les continuités écologiques » (Allag-Dhuisme et al., 2010b : 5). Ce qui revient à affirmer la dimension sociopolitique de la Trame et la place accordée aux groupes sociaux et à leurs territoires. Pour Chantal Jouanno, Secrétaire d'Etat à l'Ecologie, «L'Etat propose des orientations et une méthodologie, mais ce sont surtout les acteurs locaux qui construisent la TVB (...). Ce sont d'abord des projets de territoire $»^{2}$. Pour Serge Urbano, Vice-président de FNE, en revanche, la conception de la trame se veut plus naturaliste: "Nous sortons enfin la protection de la nature de la confidentialité. Elle doit maintenant être pleinement intégrée à l'aménagement du territoire comme critère prépondérant pour le penser et le repenser et, d'autre part, servir comme outil pour constituer l'infrastructure naturelle, la TVB qui charpentera et irriguera les territoires $»^{3}$. A travers ces différents points de vue se profilent deux conceptions de la TVB : celle qui envisage la Trame comme projet de territoire associant les habitants et les usagers et celle qui affiche une visée naturaliste prépondérante. Par-delà ces différentes conceptions, examinons à présent le dispositif mis en place par le Comité opérationnel (Comop) TVB pour tenter d'articuler les préoccupations écologiques et territoriales.

\subsection{Le dispositif TVB : entre cadrage et autonomie}

6 La TVB s'inscrit dans une suite de politiques de la nature combinant prescription et délibération (Pinton et al., 2007), mais des modifications importantes du contexte et des procédures peuvent être notées. L'initiative locale est désormais entre les mains des collectivités territoriales et de groupes rassemblés autour d'objets de nature identifiés comme patrimoine. Le recours à l'expertise est largement pratiqué et la mobilisation des connaissances (savoirs scientifiques, gestionnaires ou locaux) est favorisée par le processus de rationalisation des données (Alphandéry et Fortier, 2011). Les transformations rapides de la population des campagnes, l'effacement des agriculteurs comme groupe social majeur, la décentralisation et le rôle des ingénieries environnementales et territoriales (Janin et al., 2011) constituent aussi les éléments d'un contexte producteur de recompositions sociales. L'action publique est ainsi confrontée à une remise en cause des formats territoriaux, des normes et des pratiques, des identités collectives et individuelles. Nos travaux successifs nous permettent d'avancer que les dispositifs des politiques de la nature se sont montrés aptes à s'adapter au changement de contexte et à inclure des initiatives venues de collectifs d'acteurs ancrés sur des territoires de tailles très diverses (Pinton et al., 2007, Alphandéry et Fortier, 2007a). Ces dispositifs se présentent comme un réseau tourné vers l'action, composé d'objets hétérogènes en interrelation, et comportant des humains et des non humains, des éléments matériels et discursifs (Boussard et Maugeri, 2003).

7 Pour rendre opérationnels les grands principes adoptés dans le cadre du Grenelle, un dispositif complexe a été mis en place qui prévoit différentes échelles d'intervention 
(nationale, régionale, locale), implique une grande variété d'acteurs dans des arènes multi-niveaux, mobilise des savoirs hétérogènes, des textes de loi, des décrets d'application, etc. Concrètement, les trois niveaux d'échelles territoriales sont emboités : le niveau national vise à définir les enjeux de la Trame et à garantir la cohérence du réseau, l'échelon régional est chargé d'identifier le réseau écologique à travers le Schéma Régional de Cohérence Ecologique (SRCE) et enfin le niveau local prévoit la mise en œuvre des TVB via les projets de collectivités territoriales et les documents d'urbanisme (Plan Local d'Urbanisme, PLU ; Schéma de Cohérence Territoriale, SCoT), (Cormier et al., 2010). A travers ce dispositif, "il ne s'agit en aucune façon de porter au niveau national un cadre contraignant, descendant et ne laissant aucune marge au niveau local. Il est laissé aux territoires et à leurs acteurs toute la marge d'appréciation afin de favoriser leurs capacités d'innovation et assurer que le projet de TVB soit adapté au contexte local » (Allag-Dhuisme et al. a, 2010 : 37). Un tel dispositif fait apparaître combien le passage de l'échelle nationale ou régionale au terrain modifie la formulation des questions posées. La fonctionnalité des réseaux écologiques ne peut se concevoir en dehors d'un projet partagé par des acteurs non naturalistes. Avec ce changement d'échelle, il ne faut pas seulement intégrer d'autres savoirs sur la nature, mais aussi un projet territorial servant de cadre à des réagencements entre activités humaines, objets techniques et naturels. Cependant, ce dispositif révèle une tension entre la volonté de cadrage affichée à l'échelon national et régional, largement inspirée par des considérations naturalistes (listes d'espèces déterminantes de l'Union Internationale pour la Conservation de la Nature, UICN, habitats déterminants, zonages et inventaires déjà réalisés dans le cadre des actions publiques liées à la biodiversité) et le caractère indéterminé des mesures et des outils à mettre en œuvre au plan local. Parmi ces différents outils, on insistera tout particulièrement sur la place déterminante occupée par les connaissances dans le processus d'identification de la trame.

\subsection{Les données au cœur de la Trame}

8 La dimension cognitive constitue un élément essentiel dans les politiques publiques en faveur du vivant, comme en témoigne le recours de plus en plus fréquent à l'expertise dans la décision politique. Son importance est réaffirmée par la Stratégie Nationale pour la Biodiversité (SNB) qui encourage notamment «l'amélioration de l'expertise (...) en s'appuyant sur toutes les connaissances» et plaide en faveur d'un renforcement $d u$ "dialogue entre science et société", tout en se fixant pour objectif stratégique de "développer, partager et valoriser les connaissances" (SNB, 2011: 36). La multiplication des politiques de conservation de la biodiversité depuis les années 1990 a, en effet, généré un besoin accru de connaissances sur le patrimoine naturel et la biodiversité et a donné lieu à la mise en place d'équipements spécifiques destinés à encourager la production et la rationalisation de ces données. Au niveau européen et international, on peut mentionner la création de l'Agence Européenne de l'Environnement (AEE) au début des années 1990, la signature de la convention d'Aarhus en 1998, la promulgation de la directive Inspire en 2007. En France, l'adoption du Système d'Information sur la Nature et les Paysages (SINP) en 2007 et de l'observatoire National de la Biodiversité (ONB) en 2009, marque la volonté du Ministère de l'environnement de remédier au caractère lacunaire, disparate et difficilement mobilisable des données sur la nature et de satisfaire aux engagements européens de la France en matière d'information environnementale. L'équipement cognitif des politiques en faveur de la biodiversité s'effectue dans un contexte marqué par 
le recours croissant à des outils de plus en plus sophistiqués : Systèmes d'information géographiques, bases de données, logiciels de modélisation, etc.

L'identification du réseau écologique à l'échelle d'un territoire s'appuie sur différentes méthodes (Allag-Dhuisme et al., $2010 \mathrm{~b}$; Cemagref, 2010) qui privilégient des approches relevant de l'écologie du paysage, des approches naturalistes (entrée par espèces) ou encore des modèles de dispersion d'espèces qui permettent un travail de modélisation des corridors. Ces méthodes se distinguent par leur degré de précision, de complexité, leur capacité à confronter plusieurs sources d'information (données quantitatives mais aussi connaissances qualitatives qui prennent en compte les savoirs locaux ou de proximité). Qu'elles soient spatialisées, autrement dit fondées sur des bases de données européennes ou françaises concernant les modes d'occupation des sols (Corine Land Cover, Mos, Ecomos, etc.), qu'elles relèvent d'inventaires naturalistes ou qu'elles soient directement liées aux pratiques et aux usages de l'espace, ces différentes sources d'information permettent de se prononcer sur la qualité, la potentialité des milieux, la présence de telle ou telle espèce et les interactions entre les objets naturels et les activités humaines. Dans un contexte où les acteurs individuels ou institutionnels sont souvent confrontés à des connaissances incertaines, lacunaires et controversées, se pose alors la question de la méthode et du type de données qui sont mobilisés ainsi que celle du projet de territoire servant de cadre d'ensemble. Comment se forment les collectifs impulsant de tels projets? Quel rôle y jouent les experts et comment se combinent savoirs techniques et pratiques? Peuvent-ils devenir des savoirs partagés, mis au service d'un projet de territoire ? C'est ce que nous allons examiner à présent à partir d'un cas concret.

\section{Modélisation de la Trame Verte et Bleue au sein d'un Parc Naturel Régional}

\subsection{Qui identifie la TVB et au moyen de quels outils ?}

10 Précurseurs concernant la mise en œuvre des corridors écologiques (Girault, 2005), les PNR ont, à la suite de l'adoption de la TVB par le Grenelle de l'Environnement, tenté d'expérimenter différentes méthodes d'identification des réseaux écologiques sur leurs territoires. L'objectif était, à terme, d'engager « des actions de préservation et de restauration des corridors mais aussi des actions de requalification des infrastructures et d'aménagement de transitions entre fronts urbains, milieux naturels et espaces agricoles " (FPNRF, 2008). Nous nous intéressons ici à la méthode et aux données utilisées pour identifier les TVB à l'échelle d'un PNR ${ }^{4}$.

11 La méthodologie choisie par ce PNR combine modélisation des continuités écologiques, par un bureau d'études naturaliste, et concertation avec des experts locaux. Pour le « responsable environnement» du PNR, la modélisation apparaît aujourd'hui comme la méthode "la plus calée» permettant la production d'" une cartographie des continuités à l'échelle du parc [...] plus précise que les schémas des continuités écologiques de la région tout en utilisant des méthodes similaires, avec des SIG et en utilisant toutes les bases de données existantes, les MOS, ECOMOS, etc...». La concertation avec des experts locaux a également été privilégiée car, à l'échelle du PNR, « on voulait impliquer des acteurs du terrain, c'est prescrit par le Comop, et ça nous permettait de bénéficier des savoirs de gens qui connaissent vraiment le territoire ». L'association des deux méthodes permettait potentiellement la production de 
"cartes des continuités vraiment fiables sur le territoire avec des outils à la pointe et des connaissances de terrain».

Du point de vue de la démarche participative, le PNR a choisi de constituer des comités techniques et de pilotage regroupant «les principaux acteurs du territoire. On a essayé d'avoir les responsables des associations naturalistes, de l'ONF, des représentants des services environnement des Conseils Généraux, de la Chambre d'Agriculture aussi ». Les experts locaux contactés sont convoqués au titre des domaines d'activités qu'ils représentent et en tant qu'usagers du terrain : ils ont une légitimité en tant que professionnels et au motif qu'ils sont porteurs d'une expérience à l'échelle locale. L'objectif était de constituer des comités, plus que des groupes de travail, pouvant réagir sur les avancées des travaux du bureau d'études. Le comité technique avait pour tâches « d'accompagner et assister le bureau d'études dans les collectes des données" et le processus de modélisation. Le comité de pilotage était en charge "de valider les cartographies» produites au cours des différentes étapes de ce travail. Trois phases ont été distinguées par les experts du bureau d'études ${ }^{5}$ :

Phase 1: recueil des données jugées nécessaires à la caractérisation du territoire ${ }^{6}$, permettant l'élaboration d'une cartographie de l'occupation du sol par « compartiments écologiques ${ }^{7}$, autrement dit par type de milieux ${ }^{8}$, et l'identification des éléments fragmentant du territoire (infrastructures routières, ferroviaires, urbanisation).

Phase 2: identification et hiérarchisation de "cœurs de nature " par compartiments écologiques au travers «d'une méthode associant l'analyse de l'occupation du sol et la prise en compte de zonages liés aux milieux naturels ». Les cœurs de nature identifiés ont fait l'objet d'une validation auprès du comité de pilotage.

Phase 3: mise au jour des continuités entre "cœurs de nature " sur la base d'une modélisation des déplacements d'une espèce animale caractéristique (définie avec le comité de pilotage ${ }^{9}$ et validée par le comité technique) et d'une espèce fictive pour quatre " compartiments ». La modélisation a été effectuée à partir des cartes produites dans les phases 1 et 2 , des données disponibles sur les espèces ${ }^{10}$ et, enfin, à partir de coefficients de résistance aux déplacements ${ }^{11}$.

16 La méthodologie du projet pose cependant de multiples questions. Lors des entretiens réalisés avec différents participants et au cours des réunions des comités, nous avons observé des décalages entre les objectifs des experts du bureau d'études et ceux des membres des comités. Ces décalages portaient principalement sur la place de la modélisation et des données quantitatives associées à cet outil et sur celle des connaissances locales susceptibles d'affiner la compréhension de la fonctionnalité des Trames sur le territoire. Nous avons dès lors interrogé l'articulation de ces différents types de connaissances, qu'elles soient organisées en banques de données ou qu'elles relèvent davantage de savoirs pratiques portés par des acteurs de terrain ${ }^{12}$. Leur combinaison a-t-elle permis la production de cartographies détaillées des continuités tout en tenant compte de leur perception particulière sur le territoire?

\subsection{De la trame perçue à la trame modélisée}

17 Le bureau d'études disposait d'un large choix de connaissances sur le territoire, mais nos travaux montrent comment le recours aux savoirs biotechniques et aux bases de données officielles a été privilégié. Lors des divers entretiens que nous avons menés, certains experts locaux ont regretté une simplification des fonds cartographiques obtenus; les 
bases de données géoréférencées utilisées, par exemple, laisseraient de côté des éléments locaux voire micro-locaux essentiels à la détermination et à la hiérarchisation des continuités écologiques: "Sur les cartes [il n'] y a pas les arbres isolés, certains bosquets n'apparaissent pas (...) certains chemins, des talus et puis les roches aussi. [...] Ce sont des abris potentiels pour la faune et [ils n'] apparaissent pas sur les cartes [qu'on nous a] montrées, c'est dommage»(responsable d'une association naturaliste). Des éléments limitant la fonctionnalité de ces continuités seraient également absents des bases de données utilisées: "Ok pour prendre en compte les voies ferrées, mais [les] cartes ne nous disent pas toujours où des grillages sont installés [sur ces voies ferrées] ? Pourtant ça, ça a un sacré impact sur les déplacements des animaux!» (membre d'une institution d'aménagement des territoires).

Les cartes de déplacements des espèces produites dans le cadre de la phase 3 présenteraient également des limites. En effet, la modélisation exclut certains types de données pouvant apporter des informations nécessaires à la détermination de la présence / absence et du déplacement de ces espèces sur les compartiments identifiés. Des déplacements ont ainsi été déterminés à partir d'inventaires nationaux ou de connaissances bibliographiques générales sur les espèces mais certaines données issues de comptages locaux ont été écartées. Pour les experts du bureau d'études, ces données sont "souvent trop localisées", "indisponibles ou non mises en forme», ce qui leur a fait préférer des données globales jugées "plus pratiques et plus fiables». Toujours dans le cadre de cette phase 3, les comités et le bureau d'études n'ont pu tomber d'accord sur une espèce à modéliser pour le milieu dit « ouvert ». En effet, si le lapin, le lièvre ou encore des espèces ornithologiques étaient jugées emblématiques, localement, leurs potentialités de déplacements ne satisfaisaient pas aux exigences de la modélisation ${ }^{13}$. Seule l'espèce fictive ayant été en définitive étudiée sur ce compartiment, certains acteurs locaux ont regretté "que de telles méthodes, jugées à la pointe, ne soient pas aptes à montrer comment se déplace un lapin ou une buse » (responsable d'une association naturaliste). Le poids accordé à la modélisation, et donc l'obligation de privilégier des données formatées, n'ont pas été sans conséquence. Les cartes produites, essentiellement alimentées par des données pouvant être intégrées au SIG, n'ont pas permis une appréhension des continuités dans toute leur complexité, à l'échelle locale.

Les découpages déterminés par la modélisation ont également interpellé les experts locaux car leur "territoire complexe et diversifié » serait insuffisamment mis en valeur par le découpage en "compartiments écologiques" proposé par le bureau d'études: " D'accord pour un compartiment milieux humides mais les forêts ou les pelouses que vous classez à part, peuvent aussi être des milieux humides, comment alors modéliser le déplacement d'espèces $d u$ milieu humide si on exclut des espaces sur lesquels [ces espèces] se déplacent aussi?». Les naturalistes du bureau d'études reconnaissent les simplifications induites par la méthode mais justifient leurs regroupements par le " raisonnement scientifique suivi et mis à l'épreuve lors de précédentes réponses à des appels à projet ». Par ailleurs, ce compartimentage du territoire présente des limites : certains milieux identifiés (agricole et urbanisé) ont été écartés des travaux car jugés trop complexes à modéliser. L'exclusion de l'espace agricole a surpris certains membres du comité " pourquoi ne pas prendre en compte les champs ou les prairies qui sont des habitats à part entière? Les espèces se déplacent aussi sur les milieux agricoles!» (responsable d'une association de préservation de l'environnement). Le responsable environnement du PNR justifie cette exclusion en mentionnant un prochain appel à projet axé sur l'identification des continuités dans l'espace agricole : « On va avoir 
un deuxième appel à projet spécifiquement sur les trames vertes et l'agriculture, là on va essayer de monter un projet agri-faune avec les Fédérations de chasse. On va voir comment sur les plaines agricoles [...] on pourrait avoir des bandes enherbées le long des chemins pour avoir des milieux plus favorables à la faune chassable [...] et à tout le reste de la faune aussi ». L'actuel projet se centrerait, selon ce même responsable, davantage sur l'identification des TVB et leur possible intégration dans les documents d'urbanisme. Il semble néanmoins que soient évacués de l'analyse, sous couvert de limites méthodologiques, les espaces porteurs d'enjeux sociopolitiques où la question des continuités écologiques est source de conflits entre acteurs locaux, aménageurs et décideurs. Le parti pris d'exclure le milieu agricole n'a cependant pas fait que des mécontents. Un agriculteur, membre du comité, faisait remarquer : «les continuités, nous on ne veut pas en entendre parler sur l'espace agricole, on ne veut pas qu'on nous rajoute une couche de mesures environnementales sur le dos, on en a assez $\grave{a}$ gérer comme ça ".

En définitive, le travail de modélisation réalisé par les experts du bureau d'études comporte des limites qui n'ont pu être réduites par l'apport des connaissances de terrain des membres des comités. Ceci faisait regretter à l'un de nos informateurs «qu'on ne parte pas du terrain. Si on a ces connaissances là à l'échelle du parc à quoi bon effectuer une modélisation qui n'est pas au point! ». De nombreuses informations qualitatives n'ont pu être intégrées aux bases de données et si les membres des comités ont émis des réserves concernant la démarche du bureau d'études ou les cartes produites, ils ont validé les décisions et les documents finaux présentés par ses experts, comme si la complexité des outils utilisés ne leur avait pas permis d'être pleinement associés aux débats. Ceci révèle une asymétrie importante dans l'usage des différentes sources d'information. D'un côté le bureau d'études a valorisé une approche du territoire au travers de données quantitatives officielles globales et strictement formalisées au sein de bases de données. De l'autre, il s'est peu appuyé sur les connaissances des experts locaux qui appréhendent le territoire au travers de l'utilisation qu'ils en font au quotidien et de savoirs qualitatifs accumulés au fil d'expériences concrètes.

\section{Confrontation des savoirs : enjeux politiques et sociaux}

21 L'exemple qui précède montre à quel point l'outil de modélisation utilisé par le bureau d'études opère à la manière d'un filtre conduisant à privilégier les données quantitatives, au détriment des données qualitatives souvent considérées comme moins fiables. On voudrait à présent élargir la réflexion et revenir sur ce qui fonde le succès et la légitimité des données quantitatives en dépit des limites qu'elles comportent. Mais au-delà de cette première incursion au sein des liens qui unissent les activités cognitives et le domaine du sociopolitique, on s'interrogera sur les conditions de la prise en compte des différentes sources d'information dans le processus d'élaboration de la TVB.

Les données quantifiables constituent un instrument privilégié de mise en visibilité sur lesquels s'appuient les pouvoirs publics pour justifier le bien fondé de leurs actions. Dans son ouvrage consacré à la pratique de la statistique, A. Desrosières rappelle que celle-ci s'est développée comme outil de preuve argumentaire et comme outil de justification de la décision publique : "la mise en place de procédures de quantification est une des modalités (avec les luttes politiques et syndicales, le droit, la théorie économique, la littérature et la 
philosophie) qui contribue à faire exister socialement et à durcir quelque chose (...). Ceci n'implique pas que l'objet n'existait pas avant sa quantification. Mais celle-ci lui a donné une toute autre forme d'existence sociale » (Desrosières, 2003 : 57-58). Cette mise en visibilité par les chiffres contraste avec le côté parfois « insaisissable» des savoirs locaux inscrits dans le vécu, l'expérience et l'identité ${ }^{14}$ des personnes et souvent décriés pour la part de subjectivité qu'ils comportent. "Parce que les savoirs locaux sont considérés comme incertains, relevant davantage de l'intuition que de la raison et de l'exactitude, parce qu'ils échappent en partie à la verbalisation et donc sont difficilement accessibles, parce qu'ils sont parfois assimilés à des croyances, ils sont souvent ignorés ou font l'objet d'une faible attention. Pourtant ces savoirs sont bien réels même s'ils obéissent à d'autres codes et d'autres logiques que les savoirs scientifiques et techniques » (Fortier, 2005).

Si les chiffres incarnent une sorte d'idéal de scientificité et de rationalité, l'élaboration statistique n'est cependant pas neutre. L'anthropologue Mary Douglas montre que les grandes classifications qui ordonnent les connaissances sont les produits d'un travail institutionnel et social fort (Douglas, 1999). Les catégories statistiques relèvent de ce type de travail collectif, comme le souligne le démographe. A. Blum : «Bien-sûr la statistique a une dimension scientifique (...), mais en même temps c'est une construction dont on oublie toujours les racines, la dimension métaphorique et le sens qu'elle peut avoir. C'est une construction qui impose des catégories qui ne sont pas toujours les catégories de la pratique» (Martin, 2009). L'exercice statistique est, en effet, un processus cognitif qui amène à faire des choix, à privilégier certains aspects au détriment d'autres. En outre, la production des données s'inscrit dans une histoire et un contexte, elle correspond aux représentations sociales et politiques du moment, ce qui soulève la question de la pertinence des catégories utilisées dans le temps. Enfin, la rationalisation des données conduit à une délocalisation et à une dépersonnalisation des savoirs comme nous l'avons souligné à propos du SINP. L'emprise croissante des bases de données au nom de l'efficacité et de la transparence conduit « ̀̀ extraire les données de leur contexte social et environnemental et donc à se priver d'un certain nombre de variables souvent essentielles dans l'usage et l'interprétation de cette information" (Alphandéry, Fortier, 2011:29). Derrière les arguments de scientificité dont elle se pare, la statistique comporte un caractère réductionniste avec le risque toujours présent de naturalisation des catégories (A. Blum cité par O. Martin, 2009). Toutes ces considérations tendent à montrer qu'on ne peut considérer les données quantitatives mobilisées dans le cadre de la TVB comme des données justes, au sens où elles permettraient d'affirmer ce qui est mais comme un ensemble de connaissances partielles, socialement construites, dont la complémentarité et la pertinence doivent être débattues.

A ce stade, la donnée peut-être envisagée à travers deux dimensions qu'il est nécessaire de penser ensemble. La première, incarnée par le processus de rationalisation, tend à faire de celle-ci une information objectivée, définie de manière conventionnelle et aisément mobilisable à travers des bases de données. La seconde, en tant qu'elle reflète une diversité de pratiques, de rapports à l'espace inscrite dans un territoire donné, constitue un support de discussion entre les différents acteurs en vue d'élaborer un projet collectif. La première conception de la donnée, issue du travail de rationalisation des politiques de l'environnement, répond à un ensemble de préoccupations. Elle s'incarne notamment dans la création, au début des années 1990, de l'Agence Européenne de l'Environnement (AEE) dont l'ambition est la formation d'un espace cognitif commun destiné à rendre les données comparables d'un pays à l'autre au sein de l'UE. Le développement d'outils tels que le reporting ${ }^{15}$ ou le benchmarking ${ }^{16}$ participe de ce 
travail d'harmonisation et s'inscrit également dans une logique de contrôle et de stimulation des Etats en situant leurs performances sur des échelles normatives. Ce mouvement de standardisation répond également à la demande de transparence mise en avant par la convention d'Aarhus, au nom de l'affirmation du droit des citoyens à être informés et à s'impliquer dans l'action publique. Enfin, il va de pair avec une volonté de rationaliser l'action publique. La logique néolibérale qui émerge au tournant des années 1980 et tend à importer la logique du marché dans le domaine public s'accompagne de l'évaluation des actions publiques. Cette normalisation de la donnée s'appuie sur l'utilisation d'outils et d'instruments de plus en plus sophistiqués comme les systèmes d'information, les bases de données, les représentations cartographiques. Elle se traduit par un renforcement du pouvoir des experts, seuls à même de maîtriser ces outils et correspond à une conception singulière du politique que l'historien et philosophe Pierre Rosanvallon décrit ainsi : "Une véritable idéologie de la transparence s'est peu à peu érigée en lieu et place de l'idéal démocratique de production d'un bien commun. La transparence est devenue la vertu qui s'est substituée à l'intérêt général dans un monde dominé par l'incertitude » (Rosanvallon, 2008 : 262).

Or, dans le cas de l'élaboration de la TVB à l'échelle des territoires, c'est moins la logique de l'efficacité et de la transparence qui s'avère prépondérante que la construction d'un bien commun à travers l'élaboration d'un projet collectif. Pour reprendre les termes de Pierre Rosanvallon, «Gouverner ne consiste pas seulement, en effet, à résoudre des problèmes d'organisation, à allouer de façon rationnelle des ressources, à planifier une action dans le temps. Gouverner signifie d'abord rendre le monde intelligible, donner des outils d'analyse et d'interprétation qui permettent aux citoyens de se diriger et d'agir efficacement " (Rosanvallon, 2008 : 313). Autrement dit, il s'agit de se défaire de visions purement techniques des problèmes et d'organiser les conditions de véritables formes d'apprentissage entre les divers acteurs pour élaborer des scénarios possibles et les rendre discutables. La conservation de la biodiversité ne saurait être, comme le souligne de son côté l'écologue Patrick Blandin, une affaire d'experts mais relève avant tout de projets de sociétés. En effet, depuis la remise en cause du paradigme de «l'équilibre » en écologie, il n'est plus permis d'affirmer quel état de la nature s'impose à tous dans un endroit donné. «Ce sont bien les gens, les humains d'un lieu qui sont censés dire ce qu'ils veulent en matière d'organisation écologique et de biodiversité» (Blandin, 2009: 71). Dans cette dynamique, les experts n'incarnent plus le pouvoir, l'autorité, la vérité, mais apparaissent comme des acteurs parmi d'autres au côté des diverses parties prenantes. La mission des scientifiques, et en particulier des écologues, consiste, dès lors, toujours selon P. Blandin, à aider les acteurs locaux à bâtir des projets et à mettre en œuvre le pilotage des territoires et de la biodiversité. Ils sont appelés à mobiliser leurs compétences pour, et au service d'un véritable projet de territoire. Or c'est ici que les choses se compliquent comme le montrent les observations de terrain rapportées dans la seconde partie. La figure de l'expert animateur n'est pas aussi fréquente qu'il n'y paraît, principalement parce que la séparation classique science/politique se trouve brouillée (Joly, 2005). Cette posture suppose une certaine ouverture de la part des experts, une capacité de dialogue et en même temps une mobilisation des acteurs locaux. Dans cette optique, un travail d'animation à l'échelle du territoire de projet peut s'avérer indispensable à la création d'une dynamique collective et faciliter la médiation entre les différents acteurs et registres de connaissances. Ainsi, les expériences wallonnes de réseau écologique (Mougenot, 2003) donnent-elles une importance centrale à la production de 
connaissances intermédiaires entre les savoirs de la science et de l'expertise d'une part, les savoirs forgés à travers les pratiques et les usages locaux d'autre part.

\section{Conclusion}

L'élaboration de la TVB à l'échelle des territoires fait appel à des connaissances le plus souvent organisées en banques de données pour identifier et cartographier les espèces, les cœurs de nature et les continuités écologiques. Cette mise en visibilité de la biodiversité est l'une des fonctions du dispositif de la TVB qui contribue à faciliter l'articulation des dimensions écologiques et territoriales et joue un rôle important dans la sélection des données utilisées dans le processus d'identification de la trame. Ce dispositif s'appuie sur des structures intermédiaires dont Catherine Mougenot (2003) a analysé le rôle dans la mise en œuvre du réseau écologique wallon. Le PNR, porteur du projet dans notre étude, possède une grande partie des caractéristiques détaillées par C. Mougenot, notamment sur le plan cognitif. Situé à l'interface entre les communes et la région, il détient un savoir élargi sur son territoire et maitrise une grande partie des connaissances nécessaires à la réalisation de la trame. Nos observations centrées sur la phase initiale de l'identification des continuités écologiques ont révélé que l'implication d'un certain nombre d'acteurs de terrain au côté des experts du bureau d'études n'a pas été accompagnée d'un débat contradictoire, pas plus que n'ont été examinées d'autres options par un comité de pilotage pourtant critique sur la méthode utilisée.

Que s'est-il passé ? Seules des connaissances quantitatives ou pouvant être quantifiées ont été retenues par le bureau d'études en charge du travail de "modélisation». Une partie des membres du comité de pilotage, comme fascinés par les outils des experts ou dans l'incapacité de les discuter faute d'en avoir la maîtrise, ont dévalorisé leur propre savoir, fondé sur une familiarité avec les lieux, alors même que ce savoir le plus souvent qualitatif constitue un apport complémentaire au savoir technique et normalisé mobilisé par les experts. Nous avons ainsi pu voir comment les relations entre les diverses formes de savoir suscitaient des hiérarchisations implicites et des rapports de pouvoir. L'analyse à laquelle nous avons procédé concernant l'élaboration des trames montre comment, à chaque étape, les dimensions scientifiques, techniques, politiques et sociales ne cessent d'interagir et corroborent les analyses de Jamie Lorimer: "We cannot understand biodiversity outside of the assemblage of people, practices, technologies and other non-humans through which it is realized to us" (2006: 542). D'une manière plus générale, on peut identifier deux conceptions du savoir et du rôle des outils qui influenceront fortement par la suite les orientations du projet de Trame en construction. L'une, au nom de la science, de sa traduction en action de gestion de la nature, de l'excellence professionnelle, concerne les méthodes de plus en plus technicisées de recueil, de traitement, de mutualisation et de mise à disposition des données. L'autre, issue d'une conception pluraliste de la production des connaissances, porte l'idée qu'il faut associer tous les détenteurs de connaissances sur la nature et le territoire. La première approche correspond à la conception de la TVB centrée sur une vision à dominante naturaliste, elle se démarque d'une vision pluraliste de la trame à l'image des propos rapportés par une animatrice de PNR : «Utiliser la réflexion sur le réseau écologique pour essayer de récolter toutes les données possibles et regarder tout un territoire, c'est fédérateur et facteur de cohérence. On peut ainsi porter un nouveau regard sur son territoire, sur son paysage et donc se poser d'autres questions». Ces deux conceptions donnent à la science une place différente tout en 
questionnant le rôle des experts. Cet éclairage des enjeux liés à la construction de la TVB montre, en effet, combien les frontières entre public et privé d'une part, technique et politique de l'autre, s'avèrent aujourd'hui brouillées.

\section{BIBLIOGRAPHIE}

Allag-Dhuisme F., Amsallem J., Barthod C., Deshayes M., Graffin V., Lefeuvre C., Salles E. (coord.) Barnetche C., Brouard-Masson J., Delaunay A., Garnier CC. et Trouvilliez J., 2010a, Choix stratégiques de nature à contribuer à la préservation et à la remise en bon état des continuités écologiques - premier document en appui à la mise en oeuvre de la Trame verte et bleue en France, Proposition issue du comité opérationnel Trame verte et bleue, Paris MEEDDM ed.

Allag-Dhuisme F., Amsallem J., Barthod C., Deshayes M., Graffin V., Lefeuvre C., Salles E. (coord.) Barnetche C., Brouard-Masson J., Delaunay A., Garnier CC. et Trouvilliez J., 2010b, Guide méthodologique identifiant les enjeux nationaux et transfrontaliers relatifs à la préservation et à la remise en bon état des continuités écologiques et comportant un volet relatif à l'élaboration des schémas régionaux de cohérence écologique - deuxième document en appui à la mise en oeuvre de la Trame verte et bleue en France, Proposition issue du comité opérationnel Trame verte et bleue Paris, MEEDDM éd.

Alphandery P., Fortier A., 2007a, « A new approach to wildlife management in France. The formulation of the ORGFSH as tools for the conservation of biodiversity » Sociologia Ruralis, 47, (1), p. $42-62$.

Alphandéry P., Fortier A., 2007b, « La contestation de Natura 2000 par le « groupe des neuf » : une forme d'agrarisme anti-environnemental dans les campagnes françaises ?» in : Cornu P. et Mayaud J.-L. (dir,), Au nom de la terre. Agrarisme et agrariens en France et en Europe du 19ème siècle à nos jours, Paris : La boutique de l'Histoire, p. 427-442.

Alphandéry, P., Fortier, A., 2011, « Les associations dans le processus de rationalisation des données naturalistes ", Natures, Sciences, Sociétés, 19, p. 22-30.

Barbier J. M., 1996, « Introduction », in : Barbier J.M. (dir.), Savoirs théoriques et savoirs d'action, Paris, PUF, p. 1-17.

Berrebi-Hoffmann P., Lallement M., 2009, « A quoi servent les experts ? »Cahiers internationaux de Sociologie, Vol CXXVI, p. 5-12.

Blandin P., 2009, De la protection de la nature à la conservation de la biodiversité, Versailles, Ed. Quae, Sciences en questions.

Boussard V., Maugeri S., 2003, «Introduction », in : Boussard V., Maugeri S. (eds), Du politique dans les organisations : sociologies des dispositifs de gestion, Paris, L'Harmattan, p. 25-61.

CEMAGREF, 2010, Étude de l'intégration des continuités écologiques dans les SCOT en 2009 avant l'approbation de la loi Grenelle 2, Rapport Cemagref-MEEDDM.

Cormier L., Bernard De Lajartre A., Carcaud N., 2010, « La planification des trames vertes, du global au local : réalités et limites », Cybergeo : European Journal of Geography [En ligne], Aménagement, Urbanisme, article 504, mis en ligne le 06 juillet 2010. URL : http:// cybergeo.revues.org/23187; DOI : 10.4000/cybergeo.23187 
Demortain D., 2012, La mondialisation des experts, Paris, Ellipses.

Desrosières A., 2003, « La qualité des quantités », Courrier des statistiques, n 105-106, p. 51-63.

Douglas, M., 1999, Comment pensent les institutions, Paris, Éditions La Découverte.

Fabos J.G., Ahern J. (Ed.), 1996, Greenways : The beginning of an international movement, Elsevier

FNE, Une trame grandeur nature, Actes du $33^{\text {ème }}$ congrès de France Nature Environnement, 12-13 mars 2009.

Fortier A., 2005, « Des savoirs locaux insaisissables ? L'exemple de la tenderie aux grives en Ardenne », in : Les actions collectives pour gérer l'environnement, Vertigo, Vol 6, 3. URL : http:// vertigo.revues.org/2429

FPNRF, 2008, Arcs écologiques franciliens entre ville et campagne. Reconquête des trames éco-paysagère périurbaines, Plaquette de présentation, FPNRF \& MEEDDM.

Girault V., 2005, Mise en œuvre de corridors écologiques et ou biologiques sur le territoire des Parcs Naturels Régionaux : définition d'une méthodologie commune et recueil d'expérience, Rapport de stage, Fédération National des Parcs Naturels Régionaux de France, Consulter le fichier sur le site Janin C., Grasset D., Lapostolle D., Turquin E., 2011, L'ingénierie, signe d'intelligence territoriale? Paris, Economica.

Joly P.B., 2005, « La sociologie de l'expertise scientifique. Les recherches françaises au milieu du gué », Cahiers du GIS Risques collectifs et situations de crise, 3, mars 2005, p. 117-174.

Lorimer J., 2006, "What about the Nematodes? Taxonomic Partialities in the Scope of UK Biodiversity Conservation", Social and Cultural Geography 7(4), p. 539-558.

Martin O., 2009, «La vérité des chiffres : une illusion ? Débat entre Alain Blum, démographe, et Olivier Martin, sociologue. », La Vie des idées, 12 novembre 2009. URL : http:// www.laviedesidees.fr/La-verite-des-chiffres-une.html MEDDTL, Rapport d'étape «filière verte ». Développement de la filière " génie écologique », 30 juin 2011.

Ministère de l'écologie, du développement durable, des transports et du logement, 2011 Stratégie Nationale pour la Biodiversité 2011-2020, Paris.

Mougenot C., 2003, Prendre soin de la nature ordinaire, Paris, Editions de la MSH/INRA.

Pinton F. (dir.), Alphandéry P., Billaud J.-P., Deverre C., Fortier A., Gesniaux G., 2007, La construction du réseau Natura 2000 en France, Paris, La Documentation française.

Rémy E., Alphandéry P., Billaud J.-P., Bockel N., Deverre C., Fortier A., Kalaora B., Perrot N., Pinton F., 1999, La mise en directive de la nature. De la directive Habitats aux prémices du réseau Natura 2000. Rapport pour le ministère de l'Aménagement du Territoire et de l'Environnement.

Rosanvallon P., 2008, La contre-démocratie, Paris, Éditions du Seuil.

Stratégie Nationale pour la Biodiversité 2011-2020, Ministère de l'écologie, du développement durable, des transports et du logement, août 2011.

\section{NOTES}

1. Circulaire du ministère de l'environnement datée du 30 novembre 2009.

2. Intervention lors du Congrès de FNE à Lille, en 2009, opus cité p. 18. 
3. Ibidem, p. 59.

4. Pour des raisons de confidentialité ce PNR ainsi que les informateurs rencontrés resteront anonymes.

5. Deux chargés de projet en écologie assistés de deux sigistes et d'un cartographe.

6. À savoir le Mode d'Occupation des Sols (MOS), l'EcoMOS, le Corine Land Cover, les Schémas de Cohérence Ecologiques fournis par l'Institut d'Aménagement et d'Urbanisme d'île de France et, les Bases de Données (BD) de l'Institut Géographique Nationale (IGN): BD Topographique, Orthographique, Cartographique, Carthage ou encore les zones d'inventaires Carmen.

7. Les termes et citations entre guillemets sont issus de documents présentés en réunion par les experts du bureau d'études (présentation PowerPoint).

8. Plusieurs types de milieux ont été identifiés avec le comité technique : forestiers, à caractère humide, pelouse sèche et landes, milieu ouvert, milieu agricole, milieu urbanisé.

9. Ont été choisis : la martre des pins pour le milieu forestier, le crapaud commun pour les milieux humides, le lézard vert pour les pelouses sèches et landes. Aucune espèce significative n'a été identifiée pour le milieu ouvert.

10. Données issues de bibliographies générales, des associations naturalistes ou encore données de piégeages.

11. Un coefficient de résistance au déplacement est défini pour chaque espèce et pour chaque type d'occupation du sol. Ce coefficient reflète à la fois la difficulté que rencontre l'espèce à traverser le type de milieu concerné et l'attractivité de ce milieu pour l'espèce.

12. Les savoirs scientifiques, dans ce cas précis, renvoient à des connaissances quantitatives biologiques ou géographiques formalisées en bases de données à des échelles départementales, régionales ou nationales (entrées par espèces ou espaces naturels). Les savoirs pratiques des experts locaux sont des connaissances biologiques, géographiques ou encore agronomiques s'accompagnant d'une expérience vécue des espèces et des espaces à une échelle locale.

13. Selon les experts naturalistes du bureau d'études, le lapin est trop « peu exigeant lors de ses déplacements", le lièvre, "présent sur les secteurs de grandes cultures", est un "mauvais indicateur pour le compartiment étudié » et la méthode ne permet par la modélisation des trajectoires des espèces volantes (oiseaux ou insectes).

14. La notion de savoirs désigne tantôt des énoncés extérieurs aux individus, tantôt au contraire une réalité indissociable des agents individuels et des collectifs qui en sont les supports (Barbier, 1996).

15. Outils destinés à assurer la réalisation et la diffusion de rapports selon un format prédéterminé en vue de faciliter la communication des résultats chiffrés.

16. Terme anglais (signifiant étalonnage ou référence) qui désigne le travail de construction d'un espace d'équivalence européen, dans lequel on cherche à rapprocher les performances.

\section{RÉSUMÉS}

Le processus d'identification des continuités écologiques de la TVB donne une place importante à la mobilisation des connaissances. Il s'agit, en effet, d'articuler les dimensions écologiques et territoriales mais aussi différents types de savoirs (scientifiques, gestionnaires, locaux). Partant de l'exemple d'un travail de modélisation commandé par un Parc Naturel Régional à un bureau d'études, nous avons analysé comment les options retenues aboutissaient à se priver de 
nombreuses sources d'information locales. Réfléchissant plus globalement sur la place accordée aux données quantitatives dans un monde hanté par la performance et la transparence, cet article esquisse des pistes pour favoriser le débat et la prise en compte d'une pluralité de formes de connaissances.

The greenways identification process leaves an important place to the use of knowledge. Ecological but also territorial dimensions should be linked up along with different types of knowledge - scientific, managerial or local. Through the case study of a modeling work ordered by a Parc Naturel Regional to a research consultancy we analyzed how the chosen methods keep local sources of information away. Overall we consider the place given to the quantitative data in a world obsessed by performance and transparency. This paper outlines ways to favor discussions and to take into consideration knowledge diversity.

\section{INDEX}

Mots-clés : trame verte et bleue, normalisation, science, expertise, données quantitatives/ qualitatives, savoirs locaux, modélisation, enjeux sociopolitiques

Keywords : greenways, standardization, science, expert appraisal, quantitative/qualitative data, local knowledge, modelling, socio-political stakes

\section{AUTEURS}

\section{PIERRE ALPHANDÉRY}

Pierre Alphandéry est sociologue, à l'UMR Sadapt - INRA. Il travaille sur les processus d'écologisation du territoire et la construction de normes dans le cadre de la mise en place des politiques de conservation de la biodiversité en France. pierre.alphandery@ivry.inra.fr

\section{AGNÈS FORTIER}

Agnès Fortier est socio-ethnologue à l'UMR Sadapt - INRA. Elle travaille sur les processus d'écologisation du territoire et la construction de normes dans le cadre de la mise en place des politiques de conservation de la biodiversité en France.

\section{ANNE SOURDRIL}

Anne Sourdril, ethnologue, est chercheur à l'UMR 7533 Ladyss. Elle travaille sur les processus d'écologisation du territoire et la construction de normes dans le cadre de la mise en place des politiques de conservation de la biodiversité en France. 